\title{
How perceived threat of COVID-19 related to aggressive tendencies during the pandemic in Hubei Province and other regions of China: Mediators and moderators
}

\author{
Shichang Deng ${ }^{1} \cdot$ Xue Feng $^{2}$ \\ Accepted: 22 April 2021 / Published online: 24 May 2021 \\ (C) The Author(s), under exclusive licence to Springer Science+Business Media, LLC, part of Springer Nature 2021
}

\begin{abstract}
During the COVID-19 pandemic in early 2020, domestic violence, interpersonal conflicts, and cyberbullying have risen sharply in China. We speculate that the perceived threat of COVID-19 is related to a general, non-target-specific aggressive tendency during the pandemic. We surveyed 1556 Chinese people in April 2020 (757 people in Hubei Province, the pandemic epicenter in China, and 799 in other regions of China where the pandemic is relatively not severe). A multiple-group structural equation modeling analysis found significant total effects between perceived threat of COVID-19 and aggressive tendencies during the pandemic in both regional groups, and the effect between them was mainly achieved through the mediating roles of sense of control and powerlessness during the pandemic. For all participants, negative coping strategies significantly aggravated the association between perceived threat of COVID-19 and aggressive tendencies during the pandemic, but the buffers were different across regions of outbreak severity. For participants in other regions where the pandemic is relatively not severe, positive coping strategies could mitigate the association between perceived threat of COVID-19 and aggressions. However, for participants in Hubei Province, the epicenter of China's pandemic, higher life satisfaction was more effective in buffering. These findings extend the possible consequences of the perceived COVID-19 threat and suggest that improving the life satisfaction of residents in areas with severe outbreaks is more effective in mitigating the adverse effects of COVID-19.
\end{abstract}

Keywords COVID-19 $\cdot$ Perceived threat $\cdot$ Aggression $\cdot$ Mediator $\cdot$ Moderator

The novel coronavirus disease (COVID-19) pandemic that has swept the world since December 2019 is the most serious public health crisis since World War II (Guterres, 2020). This study paid particular attention to a prominent phenomenon during the pandemic in China: the rising aggressive tendencies among Chinese people. During the COVID-19 pandemic, people in China seemed to become restless. Domestic violence, interpersonal conflicts, and cyberbullying have risen sharply in the pandemic period (Global Times, 2020; Li et al., 2020; Ye et al., 2020). These aggressions may instigate social tensions and increase the level of social conflicts in the post-pandemic period (Censolo \& Morelli, 2020). Therefore,

Xue Feng

fengxue0716@126.com

1 School of Management, Shanghai University of International Business and Economics, Shanghai, China

2 School of Education and Sports Science, Yangtze University, Jingzhou, Hubei, China it is necessary to explore the psychological paths and intervention means of aggressive tendencies during the pandemic.

Many studies explained aggressions during the pandemic as a moral disengagement from mental stresses due to social distance, and therefore emphasize the use of positive coping strategies to mitigate aggressive tendencies (Lin, 2020; Mariani et al., 2020; Ye et al., 2020; Ying et al., 2020). However, we propose that perceived setbacks from a decreased sense of control and increased powerlessness are equally important in triggering a general, non-targetspecific aggressive tendency during the pandemic. Furthermore, in areas with high pandemic severity (e.g., Hubei Province, China, in early 2020), the buffering effect of positive coping strategies may be limited due to emotional instability brought about by ripple effects (Ahmed et al., 2020; Wen et al., 2020). Therefore, improving the life satisfaction of people in areas with severe COVID-19 pandemic outbreak through practical and material help may be a better way to reduce aggressive outcomes during the pandemic. We will test these inferences with data from a national cross-sectional survey across China. 


\section{Literature Review and Hypotheses Development}

\section{Perceived Threat of COVID-19 and Aggressive Tendencies during the Pandemic}

According to retrospective studies of the SARS pandemic (Brug et al., 2009) and the H1N1 pandemic (Bish \& Michie, 2010), perceived threat is the most immediate psychological response in emerging infectious diseases. Most early studies described individuals' perceived epidemic threat in terms of disease severity and likelihood of infection (Brewer et al., 2007). Later, Kim et al. (2016) added the dimension of protection efficacy, i.e., people's beliefs about the ability of the state or collective to protect themselves from the epidemic, to their research on responding to the threat of the Ebola epidemic. After the outbreak of the COVID-19 pandemic, Xin et al. (2020) synthesized the above concepts based on findings from China and proposed that the perceived threat of COVID-19 contains three perspectives, namely likelihood of infection, severity of the pandemic, and uncontrollability of the pandemic. Evidence from Chinese studies found that the perceived threat of COVID-19 could be influenced by a variety of factors, such as sources of pandemic-relevant information (Xin, 2020), demographic characteristics and personality (Liu et al., 2021), geographic differences (Wen et al., 2020), and stages of pandemic development (Lu \& Liu, 2020).

Aggression, which involves the intent to physically or psychologically harm, hurt, or damage, usually stems from an attitude bias or cognitive schema of strong disapproval toward others (Brodsky, 2011). Many studies have found that perceived infectious disease threat is often strongly associated with a significant increase in aggressive tendencies toward other groups (Terrizzi et al., 2013), which then lead to antisocial behaviors (Park et al., 2003), xenophobia (Faulkner et al., 2004), ethnocentrism (Navarrete \& Fessler, 2006), and prejudice (O'Shea et al., 2020). Schaller and Park (2011) proposed that the aggressive tendency during an epidemic is a behavioral immune system that keeps individuals away from suspicious others who may spread the disease. However, during the pandemic outbreak in China from December 2019, many other attacks against family members (Global Times, 2020) and doctors (Xinhua News Agency, 2020) are difficult to explain as protective behaviors activated by the behavioral immune system because these aggressions have a limited role in reducing the likelihood of disease transmission.

We speculate that during the pandemic, the perceived threat of COVID-19 may be related to a general, non-targetspecific aggressive tendency. The General Aggression Model (GAM, Allen et al., 2018) supports our speculation. The GAM explains the generation of aggression through how situation factors influence personal internal states such as cognitions, feelings, and arousal, which in turn affect aggressive or non-aggressive behavioral outcomes in appraisal and decision processes. During the COVID-19 pandemic in China, the perceived threat of COVID-19 caused a rapid increase in emotional issues (Gao et al., 2020), cognition stress (Xin et al., 2020), and arousal problems (Deng et al., 2020). As a result, people are higly likely to respond to rapidly increasing internal pressure by being aggressive. Based on the above inferences, we made the following hypothesis.

H1: The perceived threat of COVID-19 is significantly associated with aggressive tendencies during the pandemic.

\section{The Mediating Roles of Sense of Control and Powerlessness}

A sense of control is the belief in an individual's ability to shape his or her own life, which is an important factor in helping an individual adapt to changes in his or her environment (Lachman \& Weaver, 1998; Reich \& Infurna, 2017). Many studies had found that when individuals' sense of control decreased, they tend to intimidate others through aggressive behaviors in order to restore their perceived lack of control (Poon et al., 2020; Schat \& Kelloway, 2000; Sherrod et al., 1979; Warburton et al., 2016). Tong's (2004) study on stress responses of the SARS pandemic and Parmet and Sinha's (2017) study on the panic reactions of Ebola pandemic both showed that the perceived threat brought by infectious diseases could leads to a significant decrease in people's sense of control. During the COVID-19 pandemic in China, people generally experienced a low sense of control, which made their emotional state were very unstable (Qiu et al., 2020; Wang et al., 2020; Xin et al., 2020; Zhong et al., 2020). We speculate that in GAM's appraisal and decision process, low levels of sense of control make people more likely to choose aggressive behaviors to deal with their internal stress during the pandemic. Therefore, we made the following hypothesis based on the above inferences.

H2a: A sense of control mediates the association between perceived threat of COVID-19 and aggressive tendencies during the pandemic.

Powerlessness is a psychological state in which an individual feels out of control and unable to cope with current or future situations (Braga \& de Cruz, 2009). It is also a pervasive negative psychological outcome during the COVID-19 pandemic in China (Biddlestone et al., 2020; Guo et al., 2020; Liu et al., 2020). Powerlessness can be seen as a frustrating experience. The Frustration-Aggression Hypothesis suggests that aggressiveness is often related to the driving forces brought about by a state of frustration: if one is unable to 
counteract the source of frustration, then his or her aggressiveness may get displaced onto an innocent targe (Berkowitz, 1989). We speculate that in GAM's appraisal and decision process, the powerless state during the pandemic increases people's likelihood to vent their aggressive tendencies out on others. We made the following hypothesis based on the above reasoning.

H2b: A powerlessness mediates the association between perceived threat of COVID-19 and aggressive tendencies during the pandemic.

\section{The Moderating Roles of Coping Strategies and Life Satisfaction during the Pandemic}

Coping strategies are psychological methods that individuals use to manage their thoughts, feelings, and actions during frustrating and stressful events (Skinner \& ZimmerGembeck, 2016). Coping strategies could be categorized as positive (e.g., try to solve the current situation) or negative (e.g., escape through alcohol). Many studies have shown that positive coping strategies can help individuals reduce aggressive tendencies in frustration, while negative coping strategies are significantly associated with increased aggression (Dempsey, 2002; Maxwell \& Siu, 2008). Therefore, we posit that positive coping strategies during the pandemic made individuals less likely to aggressive outcomes in GAM's appraisal and decision process, but negative coping strategies during the pandemic done the opposite. We made the following two hypotheses based on the above inferences.

\section{H3a: Positive coping strategies mitigate the effect of the perceived threat of COVID-19 on aggressive tendencies during the pandemic. \\ H3b: Negative coping strategies exacerbate the effect of the perceived threat of COVID-19 on aggressive tenden- cies during the pandemic.}

The Chinese government has taken many measures to ensure people's daily life during the pandemic (Xinhua News Agency, 2020), however, the overall life satisfaction of the Chinese population declined rapidly during the pandemic due to the perceived threat of COVID-19 (Lin, 2020; Zhang et al., 2020c). Various studies shown that a higher level of life satisfaction could reduce the effect of adverse events on aggressions (Orzechowska et al., 2009; Suldo \& Huebner, 2004; Valois et al., 2001; Velotti et al., 2017). Thus, we propose that if people's life satisfaction is relatively high during the pandemic, they may be less likely to generate aggressive thoughts. We made the following hypothesis based on the above reasoning.
H3c: A relatively high life satisfaction level during the pandemic buffers the effect of the perceived threat of COVID-19 on aggressive tendencies during the pandemic.

\section{The Different Moderating Effects between People in Hubei Province and Other Regions of China}

Hubei Province was the epicenter of the COVID-19 outbreak in China in early 2020, which accounted for more than $79 \%$ China's total cases during the pandemic (Xinhua News Agency, 2020). Therefore, it is necessary to consider whether there are differences between people in Hubei Province and other regions of China in the above three sets of research hypotheses.

Our $\mathrm{H} 1$ (the perceived threat of COVID-19 is significantly associated with aggressive tendencies during the pandemic) and $\mathrm{H} 2$ (sense of control and powerlessness mediate the association between perceived threat of COVID-19 and aggressive tendencies during the pandemic) are inferred from general psychological theories and research evidence. Therefore, we believe these two research hypotheses have general applicability. For this reason, although people in Hubei Province may have significantly higher values of these variables than people in other regions of China, we speculate that there are no differences between the two groups of people in terms of the relationship between these variables.

However, in H3 (moderation roles of coping strategies and life satisfaction), there may be differences among people in Hubei Province and other regions of China. Wen et al. (2020) and Ahmed et al. (2020) found that people's mental states showed a ripple effect in regions with different pandemic severity, i.e., people in Hubei Province (the epicenter of the outbreak) exhibited a higher level of perceived COVID-19 threat and more emotional instability. Many studies have shown that when emotional instability increases, the role of positive coping strategies will be limited (Mariani et al., 2020; Ogueji et al., 2021; Wasil et al., 2021). Therefore, we hypothesized that:

H4: For people in Hubei Province (the epicenter of the COVID-19 outbreak in China), the moderation role of positive coping strategies will no longer be significant.

\section{The Current Study}

We summarized our hypotheses as a conceptual model in Fig. 1. In sum, this study aims to explore the relationship between perceived threat of COVID-19 and aggressive tendencies during the pandemic, and the mediating roles of sense 


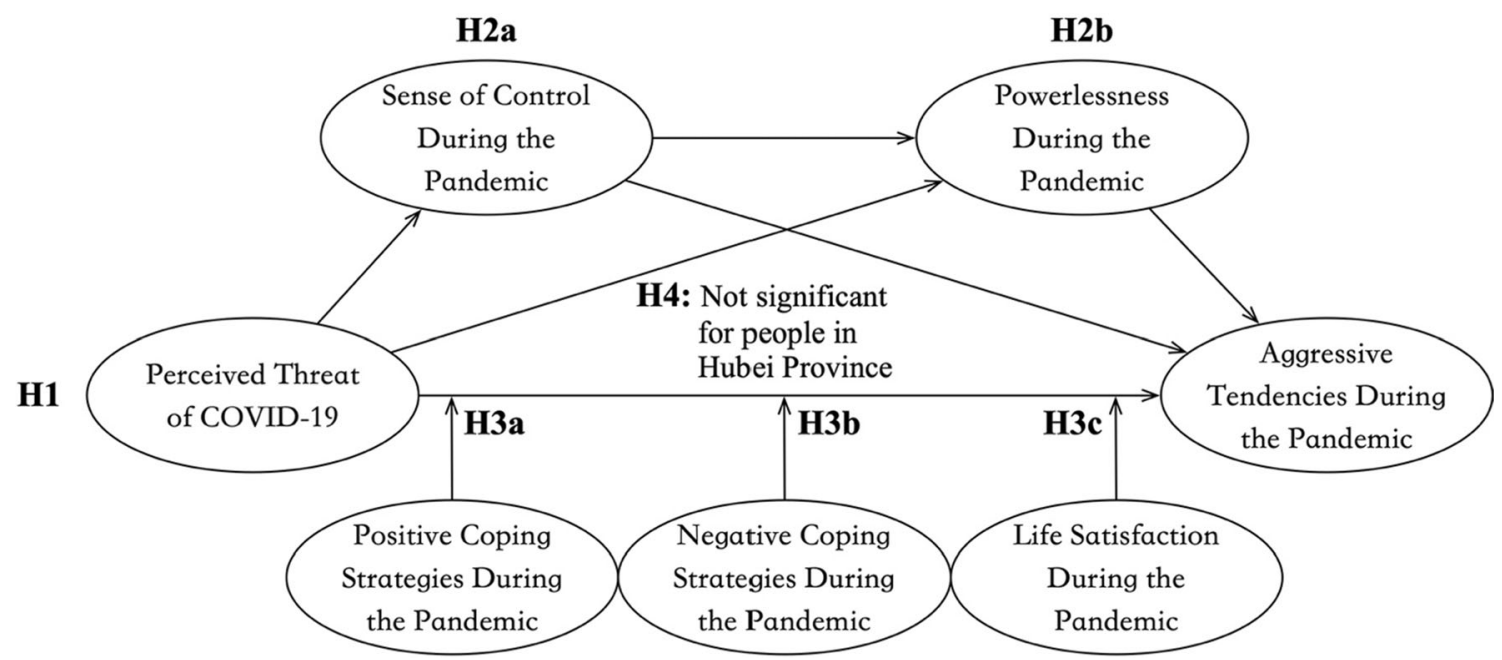

Fig. 1 The conceptual model of this study

of control and powerlessness, as well as the moderating roles of coping strategies and life satisfaction. In order to test these hypotheses, we conducted a survey at the end of April 2020. At that time, the outbreak in China was just about under control (Xinhua News Agency, 2020), so people could thoroughly reconsider their mental states during the pandemic since the outbreak. By providing first-hand survey data from the country that the pandemic firstly outbreaks on a large-scale, we hope the findings may deepen the understanding of the public's mental appraisal processes in the early stages of COVID19 outbreak and provide a scientific reference to policymakers to develop better and provide psychological assistance and social services in an emergent public health crisis.

\section{Method}

\section{Procedure}

A cross-sectional design survey with a national sample was conducted. Questionnaires were distributed through a Chinese online survey platform on April 26, 2020 and were valid for three days. Participants across China were free to decide whether or not to take this survey for a small monetary reward. On the first page of the survey, an information sheet describing the study objectives and procedures was presented to participants, telling them that their IP address would be recorded to prevent duplicate responses (an ostensible reason) and that if they did not agree, they would not be allowed to participate in the survey. This survey has been pre-registered in and ethically approved by the Department of Psychology of Yangtze University (Hubei, China).

We used IP addresses to determine where participants lived during the pandemic. Since travel controls were still in place in most parts of China at the time of the survey (Xinhua News Agency, 2020), the location of the IP address was basically where the participants actually lived from February to April 2021, during the height of the pandemic in China. We also asked participants "where have you mainly lived from February 2020 to now?" Participants whose answers do not match their IP address were excluded manually.

\section{Participants}

By April 29th, 2020, 1556 people effectively responded to all items. Their demographics are shown in Table 1.

\section{Measures}

Independent Variable We used Xin et al. (2020)'s 13-item Perceived Threat of COVID-19 Scale to assess the samenamed variable. This scale contains three subscales: Likelihood of Infection (e.g., "I feel that if I am not careful, my family or myself are very likely to infected by the coronavirus"); Severity of the Pandemic (e.g., "I feel that the current situation of the pandemic is very serious"); and Uncontrollability of the Pandemic (e.g., "I feel that it is tough to control the pandemic effectively"). Participants responded on a Likert scale from 1 (very much disagree) to 5 (very much agree). A higher score indicates a higher level of perceived threat of COVID-19. In this study, the Cronbach's $\alpha$ of this scale was 0.88 .

Dependent Variable The 12-item Short-Form Buss-Perry Aggression Questionnaire (Diamond \& Magaletta, 2006) was used to assess the general aggressive tendencies. Items of this questionnaire were grouped into four subscales: Physical Aggression (e.g. "given enough provocation, I may hit another 
Table 1 Demographics of participants

\begin{tabular}{llll}
\hline & $\begin{array}{l}\text { Participants in } \\
\text { Hubei province }\end{array}$ & $\begin{array}{l}\text { Participants in other } \\
\text { regions of China }\end{array}$ & 1556 \\
\hline$N$ & 757 & 799 & $26.76(6.76)$ \\
$M_{\text {age }}(S D)$ & $26.36(7.23)$ & $27.14(6.26)$ & 786 \\
Sex: Female & 402 & 384 & 770 \\
Male & 355 & 415 & 575 \\
Occupation: Student & 335 & 240 & 746 \\
Have a formal job & 291 & 455 & 61 \\
Have a part-time job & 27 & 34 & 138 \\
Freelancers & 76 & 62 & 36 \\
Unemployed & 28 & 8 & 980 \\
Marital Status: Single & 506 & 474 & 364 \\
Married & 242 & 3 & 12 \\
Separated or divorced & 9 & &
\end{tabular}

person"); Verbal Aggression (e.g., "I can't help getting into arguments when people disagree with me"); Anger (e.g., "sometimes I fly off the handle for no good reason"); and Hostility (e.g., "I feel I have gotten a raw deal out of life"). The term "during the pandemic" was added before each item to measure participants' aggressive tendencies during the pandemic period. Participants responded on a Likert scale from 1 (very much disagree) to 5 (very much agree) A higher score indicates higher aggressive tendencies during the pandemic. In this study, the Cronbach's $\alpha$ of all 12 items was 0.94 .

Mediating Variables The 12-item Sense of Control Scale (Lachman \& Weaver, 1998) was used to assess the samenamed variable. This scale contains two subscales: Personal Mastery (e.g., "I believe my future depends on me") and Perceived Constraints (e.g., "I feel things beyond my control"). The term "during the pandemic" was added before each item to evaluate participants' sense of control during that period. Participants responded on a Likert scale from 1 (very much disagree) to 5 (very much agree). We reverse-scored items of perceived constraints subscale, so for the whole scale, a higher score indicates a higher sense of control level during the pandemic. In this study, the Cronbach's $\alpha$ of all 12 items was 0.94 .

Braga \& Cruz's (2009) 12-item Powerlessness Assessment Tool was used to assess the same-named variable. Items of this scale were grouped into three subscales: Powerlessness in Perform Behavior (e.g., "I feel incapable of looking after myself"); Powerlessness in Make Decision (e.g., "Nothing I do can change the situation I am in"); and Powerlessness in Control (e.g., "I feel sad when I think I need someone to help me"). The term "during the pandemic" was added before each item to measure participants' powerlessness during that period. Participants responded on a Likert scale from 1 (very much disagree) to 5 (very much agree). A higher score indicates higher powerlessness during the pandemic. In this study, the Cronbach's $\alpha$ of this scale was 0.91 .

Moderating Variables The 20-item Simplified Coping Style Questionnaire, which was culturally adapted by Xie (1998) based on the Ways of Coping Questionnaire (Folkman \& Lazarus, 1988), was used to assess the same-named variable. Items of this questionnaire were grouped into Positive Coping Strategies (e.g., "I relief through work, study or other similar activities") and Negative Coping Strategies (e.g., "I often imagine that some miracle might happen to change things"). The term "during the pandemic" was added before each item to evaluate participants' coping strategies during that period. Participants responded on a Likert scale from 1 (very much disagree) to 5 (very much agree). A higher score of a subscale indicates a higher tendency to adopted corresponding coping strategies during the pandemic. In this study, the Cronbach's $\alpha$ s were 0.88 for the Positive Coping Subscale and 0.71 for the Negative Coping Subscale, respectively.

The 5-item Satisfaction With Life Scale (Pavot \& Diener, 1993) was used to assess the same-named variable. The term "during the pandemic" was added before each item to evaluate participants' life satisfaction during that period, such as "During the pandemic, my life is close to my ideal in most ways." Participants responded on a Likert scale from 1 (very much disagree) to 5 (very much agree). A higher score indicates a higher life satisfaction during the pandemic. In this study, the Cronbach's $\alpha$ of all 5 items was 0.82 . 


\section{Statistical Methods}

We tested the normality of all surveyed variables. Skewness of all variables were ranged from -0.49 to 0.46 , and Kurtosis ranged from -0.48 to 0.37 , and all $p$ were $<0.001$ in the Shapiro-Wilk test. The results showed that the distributions of all variables slightly violated the assumption of normality (see Appendix 1).

Therefore, we used nonparametric approaches to analyze all variables. First, the Mann-Whitney U test was used to explore the possible difference of all variables between the two groups of participants (from Hubei Province or other parts of China). Then, Spearman's Rho correlation was used to examine the preliminary relationships between variables.

Next, a multiple-group Structural Equation Modelling (SEM) was used to test the mediation effects, as well as the possible difference of the mediation effects between two groups of participants in Hubei Province or other parts of China. We used the items of each scale as observed variables for their respective latent variables. Li (2016) suggested that when observed variables in latent variable models were ordered categorical data, a Unweighted-Least-Squares (ULS) estimation methods could achieve more reliable results. Therefore, the ULS estimation method was used in our SEM analysis. We investigated the goodness of fit for the proposed model based on the work of $\mathrm{Hu}$ and Bentler (1999), where the cutoff values for the goodness-of-fit index (GFI), comparative fit index CFI), normed fit index (NFI), and Tucker-Lewis Index (TLI) were > 0.95 ; RMSEA were $<0.06$; SRMR were $<0.08$. Although the above cutoffs only concerned continuous data that using the normality-based Maximum Likelihood (ML) estimation, but Xia and Yang (2019) suggested that applying the conventional cutoffs to the ULS estimation is reasonable.

Finally, the effects of moderation variables were examined by Generalized Linear Models (GLM). GLM is a flexible regression method that allows variables to have distributions other than normal (Myers \& Montgomery, 1997).

The freeware R's lavaan package (v0.6-7, Rosseel, 2012) completed the SEM analysis, and the freeware jamovi (v1.6, The jamovi project, 2021) completed the other analyses.

\section{Results}

Mann-Whitney U test showed that participants in Hubei Province were significantly higher than that in other regions of China on the variables of perceived threat of COVID-19, aggressive tendencies during the pandemic, and sense of powerless during the pandemic. However, sense of control during the pandemic, positive coping during the pandemic, and life satisfaction during the pandemic were significantly lower for participants in Hubei Province than for participants in other regions of China, see Appendix 2. This indicated that the COVID-19 pandemic in early 2020 had greater psychological impacts on the residents of Hubei Province (the epicenter of the outbreak). Therefore, it is necessary to analyze the two groups of participants in different regions separately.

Spearman's Rho correlations were calculated separately for the variables between participants in Hubei Province and those in other regions of China, see Table 2. Overall, the correlations between participant were similar across regions. Aggressive tendencies during the pandemic were generally significantly positive related to perceived threat of COVID-19, sense of powerless during the pandemic, and negative coping during the pandemic, but were generally significantly negative related to sense of control during the pandemic, positive coping during the pandemic, and life satisfaction during the pandemic. These correlations were consistent with what we have speculated in hypotheses.

We use a multiple-group Structural Equation Modelling (SEM) to test H1, H2a and H2b. Based on Hayes (2013) Model 6 ( 2 mediators), our model contained both observed variables (item of each scale) and latent variables, see Fig. 2. To explore possible differences between participants in Hubei Province and other regions of China, a multiple-group analysis was conducted by a ULS estimation. The model's goodness-of-fits were acceptable $(\mathrm{GFI}=0.99, \mathrm{CFI}=0.96$, $\mathrm{NFI}=0.95, \mathrm{TLI}=0.96, \mathrm{RMSEA}=0.08, \mathrm{SRMR}=0.07$ ). Although the RMSEA slightly exceeds the cutoff $(0.06)$ suggested by Hu and Bentler (1999), Shi and Maydeu-Olivares (2020) found that estimation methods had substantial impacts on the RMSEA. They suggested that SRMR is robust across estimation methods, so, the same criterion can be applied when using the SRMR to evaluate model fit, regardless of the choice of estimation method. Therefore, it can be considered that our model fit is acceptable.

For participants in Hubei Province and those in other regions of China, the model explains $40.5 \%$ and $45.9 \%$ of $a g$ gressive tendencies during the pandemic $\left(R^{2}=0.405\right.$ and $0.459)$, respectively. Table 3 decomposed the effects between perceived threat of COVID-19 and aggressive tendencies during the pandemic. For all participant, there were significant total effects between these two variables, but the total effect was slightly higher for participants in Hubei Province than for other regions in China. The direct effect between perceived threat of COVID-19 and aggressive tendencies during the pandemic was very small (below the absolute value of 0.03), as well as the indirect effect through powerlessness only (below 0.04). The results suggested that the association between perceived threat of COVID-19 and aggressive tendencies 
Table 2 Spearman's Rho correlations between variables

\begin{tabular}{|c|c|c|c|c|c|c|c|}
\hline & 1 & 2 & 3 & 4 & 5 & 6 & 7 \\
\hline 1 Perceived threat of COVID-19 & - & $0.21 * * *$ & $-0.35 * * *$ & $0.28 * * *$ & 0.07 & $0.10 * *$ & $-0.11 * *$ \\
\hline 2 Aggressive tendencies during the pandemic & $0.22 * * *$ & - & $-0.59 * * *$ & $0.62 * * *$ & $-0.33 * * *$ & $0.25 * * *$ & $-0.32 * * *$ \\
\hline 3 Sense of control during the pandemic & $-0.45^{* * *}$ & $-0.52 * * *$ & - & $-0.69 * * *$ & $0.19 * * *$ & $-0.24 * * *$ & $0.36^{* * *}$ \\
\hline 4 Sense of powerless during the pandemic & $0.32 * * *$ & $0.57 * * *$ & $-0.62 * * *$ & - & $-0.39 * * *$ & $0.30 * * *$ & $-0.34 * * *$ \\
\hline 5 Positive coping during the pandemic & $0.15^{* * *}$ & $-0.17 * * *$ & 0.03 & $-0.19 * * *$ & - & $0.17 * * *$ & $0.41 * * *$ \\
\hline 6 Negative coping during the pandemic & $0.18^{* * *}$ & $0.30 * * *$ & $-0.33 * * *$ & $0.36^{* * *}$ & $0.28 * * *$ & - & 0.04 \\
\hline 7 Life satisfaction during the pandemic & $-0.12 * * *$ & $-0.27 * * *$ & $0.33 * * *$ & $-0.21 * * *$ & $0.36^{* * *}$ & $0.11 * *$ & - \\
\hline
\end{tabular}

Below the diagonal are the participants in Hubei Province and above the diagonal are the participants in other regions of China. $* * p<0.01, * * *$ $p<0.001$

during the pandemic was mainly achieved through the mediation roles of sense of control, and sense of control and powerlessness in serial.

In a Generalized Linear Model (GLM), set aggressive tendencies during the pandemic as the dependent variable, set perceived threat of COVID-19, positive coping strategies, negative coping strategies, and life satisfaction as covariates. All variables except dependent variables were centralized. Among the participants in Hubei Province, we found significant interactions between perceived threat of COVID-19 and negative coping strategies ( $\mathrm{est}=-0.22, \mathrm{SE}=0.06, p<0.001$ ) and life satisfaction (est $=-0.12, \mathrm{SE}=0.05, p=0.014$ ), but the interaction with positive coping strategies did not reach a significant level (est $=0.00, \mathrm{SE}=0.07, p=0.962$ ). Among the participants in other regions of China, however, the interactions between perceived threat of COVID-19 and positive coping strategies (est $=-0.15, \mathrm{SE}=0.06, p=0.013$ ) and negative coping strategies ( $\mathrm{est}=0.12, \mathrm{SE}=0.05, p=0.017$ ) were significant, but the interaction with life satisfaction did not reach a significant level (est $=0.04, \mathrm{SE}=0.04, p=0.372$ ).

For both participants in Hubei Province and those in other regions of China, a high level of negative coping strategies (+1 SD) made the perceived threat of COVID19 generate more aggressive tendencies during the pandemic. However, there are regional differences in the variables that buffer this effect. For participants in Hubei Province, a high level of life satisfaction (+1 SD) mitigated the above adverse effect, but the moderating role of positive coping strategies was not significant. For participants in other regions of China, a high level of positive coping strategies $(+1 S D)$ buffered the impact of perceived threat of COVID-19 on aggressive tendencies during the pandemic, but the moderating role of life satisfaction failed to reach a significant level. See Fig. 3.

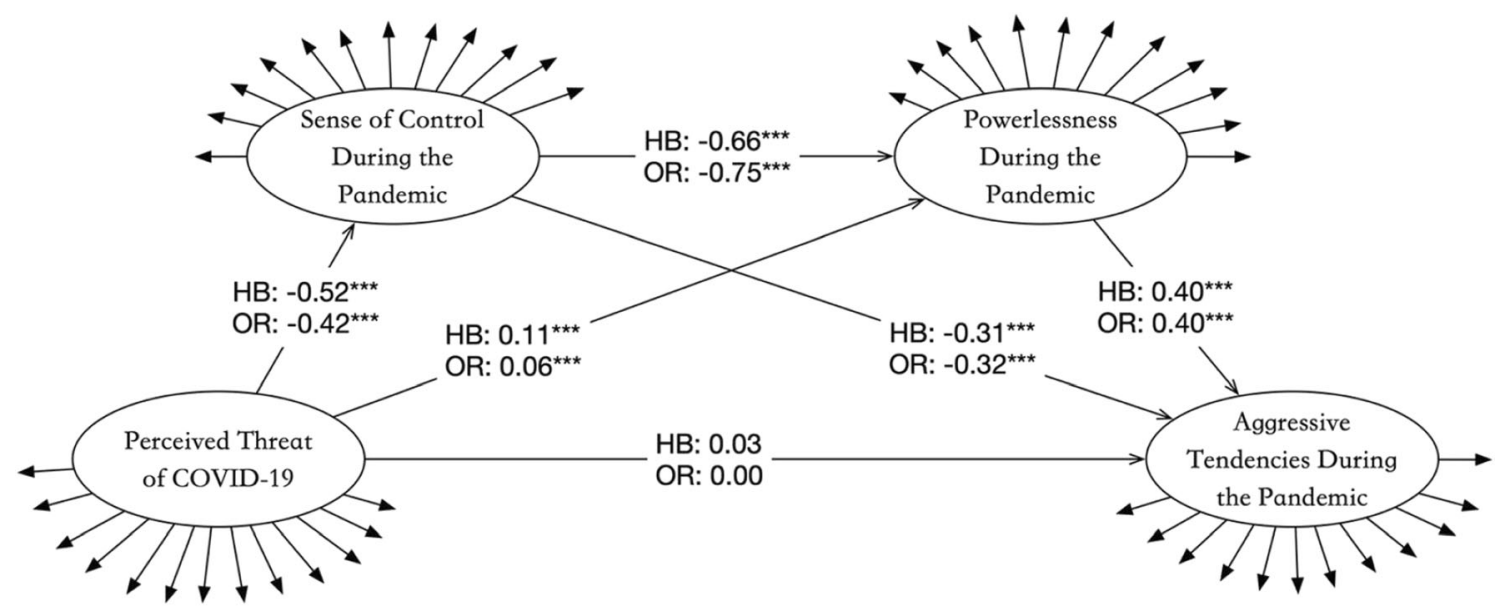

Fig. 2 Perceived threat of COVID-19 related to aggressive tendencies during the pandemic through the mediating roles of sense of control and powerlessness. HB is participants in Hubei Province, and OR is

participants in other regions of China. $* * p<0.01$, *** $p<0.001$. The extension arrows of latent variables represent the item of each scale. See supplementary material for the coefficients of the measurement model 
Table 3 Effects between perceived threat of COVID-19 and aggressive tendencies during the pandemic

\begin{tabular}{|c|c|c|c|c|c|c|}
\hline Effects & Participants in & Std.Est & SE & $p$ & Lower $95 \%$ CI & $\begin{array}{l}\text { Upper } \\
95 \% \text { CI }\end{array}$ \\
\hline \multirow[t]{2}{*}{ Direct effect } & Hubei Province & 0.03 & 0.01 & $<0.001$ & -0.05 & -0.01 \\
\hline & Other Regions of China & 0.00 & 0.01 & 0.919 & -0.02 & 0.02 \\
\hline \multirow[t]{2}{*}{ Indirect effect though sense of control only } & Hubei Province & 0.16 & 0.01 & $<0.001$ & 0.15 & 0.18 \\
\hline & Other Regions of China & 0.14 & 0.01 & $<0.001$ & 0.12 & 0.15 \\
\hline \multirow[t]{2}{*}{ Indirect effect through powerlessness only } & Hubei Province & 0.04 & 0.00 & $<0.001$ & 0.04 & 0.05 \\
\hline & Other Regions of China & 0.02 & 0.00 & $<0.001$ & 0.01 & 0.03 \\
\hline \multirow[t]{2}{*}{ Indirect effect though sense of control and powerlessness in serial } & Hubei Province & 0.14 & 0.00 & $<0.001$ & 0.13 & 0.14 \\
\hline & Other Regions of China & 0.13 & 0.01 & $<0.001$ & 0.11 & 0.14 \\
\hline \multirow[t]{2}{*}{ Total effect } & Hubei Province & 0.31 & 0.01 & $<0.001$ & 0.30 & 0.32 \\
\hline & Other Regions of China & 0.29 & 0.01 & $<0.001$ & 0.27 & 0.30 \\
\hline
\end{tabular}

Std.Est stands for Standardized Estimate, CI stands for Confidence Interval

\section{Discussion}

\section{Perceived Threat of COVID-19, Aggressive Tendencies during the Pandemic, and Mediators between Them}

H1 assumed that the perceived threat of COVID-19 is significantly associated with aggressive tendencies during the pandemic. As $\mathrm{H} 1$ described, the results showed a significant positive total effect between the above two variables. Moreover, the direct effect size between them was relatively small. As $\mathrm{H} 2 \mathrm{a}$ and $\mathrm{H} 2 \mathrm{~b}$ hypothesized, sense of control and powerlessness during the pandemic mediated the effect between perceived threat of COVID-19 and aggressive tendencies during the pandemic.

Our findings echoed other independent similar studies. A national survey in China conducted by Li et al. (2020) in February 2020 found an increased trend of aggression (2.70 out of 5). Ying et al. (2020) interpreted the increasing in aggression during the pandemic as a result of excess psychological energy brought about by social distancing and life conflicts. Based on Chinese sample, Ye et al. (2020) also found that the fear of COVID-19 was significantly related to online aggressive behavior during the pandemic. They use moral disengagement to explain this phenomenon and suggest that family cohesion could act as a buffer.

Our research suggests that in addition to life conflicts and moral issues, a decline in sense of control and an increase in powerlessness also associated with the rise in aggressions during the COVID-19 pandemic. The perceived threat of COVID-19 caused much stress, which significantly impacted people's internal states (Xin et al., 2020). The General
Aggression Model (GAM) posits that individuals are highly likely to deal with their internal stress through aggressive behaviors in the appraisal process. Traditional researches on GAM suggests that individuals make aggressive decisions when they believe they are capable enough (Allen et al., 2018). Our study found that a perceived lack of competence in the pandemic (decreased sense of control and increased powerlessness) also makes individuals generate aggressive outcomes. The results of this study provide a reference for follow-up studies on aggressions during the COVID-19 pandemic.

\section{Different Buffers among Participants from Hubei Province and Other Regions of China}

H3a assumed that positive coping strategies mitigate the effect of the perceived threat of COVID-19 on aggressive tendencies during the pandemic, and $\mathrm{H} 4$ hypothesized that for people in Hubei Province (the epicenter of the COVID-19 outbreak in China), the moderation role of positive coping strategies would be limited. The results support the above two hypotheses. It is found only among participants from other regions of China that positive coping strategies can play a significant moderation role between the perceived threat of COVID-19 and aggressive tendencies during the pandemic.

$\mathrm{H} 3 \mathrm{c}$ assumed that a relatively high life satisfaction level during the pandemic could buffer the effect of the perceived threat of COVID-19 on aggressive tendencies during the pandemic. However, this hypothesis is only partially supported by the result. We found that only among Hubei Province participants, life satisfaction can buffer the effect between the perceived threat of COVID-19 


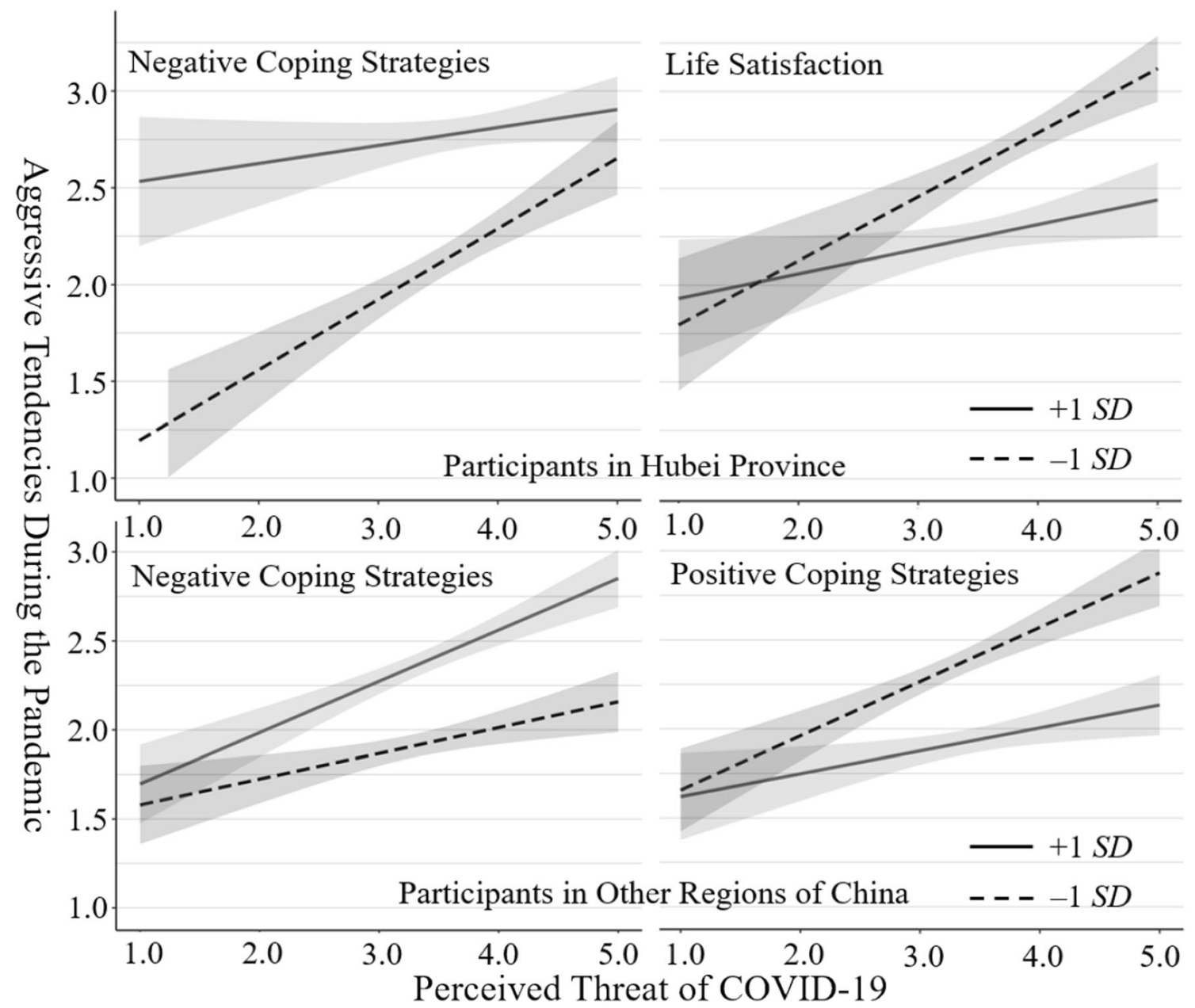

Fig. 3 Moderation effects of positive coping strategies, negative coping strategies, and life satisfaction between perceived threat of COVID-19 and aggressive tendencies during the pandemic. Shadows are $95 \%$ Confidence Intervals

and aggressive tendencies during the pandemic. Only $\mathrm{H} 3 \mathrm{~b}$ (negative coping strategies exacerbate the effect of the perceived threat of COVID-19 on aggressive tendencies during the pandemic) was fully supported, demonstrating that for both participants from Hubei Province and other regions of China, negative coping strategies generally aggravated the relationship between perceived threat of COVID-19 and aggressive tendencies during the pandemic.

We speculate that this phenomenon is caused by the ripple effect of different severity of the COVID-19 pandemic. Hubei Province was the epicenter of the 2020's COVID-19 outbreak in China. At the time of this study, Hubei Province accounted for more than $79 \%$ of the country's confirmed cases (Xinhua News Agency, 2020). Many studies have found a ripple effect in
China's 2020 COVID-19 outbreak (Wen et al., 2020; Zhang et al., 2020a; Zhang et al., 2020b), suggested that the closer to the epicenter makes stronger stress and emotional reactions. For people in other regions of China where the pandemic is relatively not severe, positive coping strategies could mitigate the adverse effect of COVID19. However, for people in the epicenter (Hubei Province), mere psychological coping strategies alone were not enough in alleviating the impact of the pandemic. People at the epicenter need a definite increase in life satisfaction to cope with the impact of COVID-19.

As for the insignificant moderating role of life satisfaction in the relationship between the perceived threat of COVID-19 and aggressive tendencies among participants from other regions of China, we suggest that this is influenced by the participants' high life satisfaction 
level. Many studies have pointed out that a decrease in life satisfaction is one of the important reasons for triggering aggression outcomes (e.g., MacDonald et al., 2005; Valois et al., 2001). Since the COVID-19 outbreak in China in early 2020 was mainly concentrated in Hubei Province, the life satisfaction of residents in Hubei declined rapidly, while the life satisfaction of residents in other regions of China remained at normal levels. Therefore, when there is no significant decrease in life satisfaction, this variable's moderating effect on the relationship between the perceived threat of COVID19 and aggressive tendencies during the pandemic becomes less pronounced.

\section{Theoretical and Practical Implications}

The theoretical contribution of this study is to extend the possible behavioral consequences of perceived threats of COVID-19. During the COVID-19 pandemic, the perceived threat of the pandemic attracted a great deal of attention from researchers in China and other countries of the world. At present, studies on threat perception of COVID-19 are mainly focused on its concern with economic confidence (Xin, 2020), anxiety reaction (Wen et al., 2020), pandemic-control confidence (Dryhurst et al., 2020), variety-seeking (Kim, 2020), and postpandemic consumption willingness (Deng et al., 2020). This study showed that the perceived threat of COVID19 may related to a universal, non-target-specific aggressive tendency, provides a reference from first-hand data for subsequent studies.

The practical contribution of this study is that life satisfaction should be guaranteed for people in areas where the pandemic is severe. For all people, negative coping strategies (such as smoking, drinking, and fantasy) cannot solve problems; on the contrary, these negative coping strategies aggravated the association between perceived threat of COVID-19 and aggressive tendencies during the pandemic. Positive coping strategies (such as positive thinking, seeking positive hobbies, and communicating with others) could counteract the negative effects of COVID-19 to some extent, but they do not always work. For areas with a relatively low pandemic severity, positive coping strategies do have some effect. However, for areas with a high pandemic severity, the role of positive coping strategies failed to reach a significant level. We found that compared with psychological regulations, life satisfaction can help people in regions of severe outbreak (such as Hubei Province) to cope with the negative effects of COVID-19. This is not to say that positive psychological coping strategies are not important to people in Hubei Province, but that when the pandemic is serious, increasing epicenter residents' overall life satisfaction level through practical and material help are more effective in buffering the adverse effects of COVID-19.

\section{Limitations and Directions for Future Studies}

There are three limitations to this study. First, we only conducted a cross-sectional survey and analyzed relationships across questionnaire data, which cannot provide substantial causality evidence. Moreover, we only measure people's aggressive tendencies instead of behavior data of aggressions. Future studies better adopt experimental methods to investigate the causality of COVID-19 threats and aggressive behaviors.

Second, we did not rule out the influence of regional culture on aggressive tendencies. China has a complex regional culture, studies believe that Hubei people may more aggressive (Brown, 2001). Future studies may include more control variables and employ more refined research designs to explore aggressions during the pandemic.

Third, our data are cross-sectional, so it remains debatable whether our findings can be applied to all stages of the pandemic. We recommend that future studies consider a time-series or longitudinal study design to gain greater explanatory power.

\section{Conclusion}

Through a national cross-sectional survey at the end of April 2020 (when the COVID-19 pandemic was just being effectively controlled in China), we found that there is a significant positive association between perceived threat of COVID-19 and aggressive tendencies during the pandemic. The effect between them is mainly achieved through the mediating roles of sense of control and powerlessness during the pandemic.

Negative coping strategies generally aggravated the effect of perceived threat of COVID-19 on aggressive tendencies during the pandemic, but there are regional differences in buffers between them. For participants in other regions of China where the pandemic is relatively not severe, positive coping strategies could buffer the effect between perceived threat of COVID-19 and aggressive tendencies during the pandemic. However, for participants in Hubei Province, the epicenter of China's pandemic, higher life satisfaction was more effective in mitigating the adverse effects of COVID-19. 


\section{Appendix 1}

Table 4 Normality test of all variables

\begin{tabular}{llllll}
\hline & Skewness & $\begin{array}{l}\text { Std. error } \\
\text { skewness }\end{array}$ & Kurtosis & $\begin{array}{l}\text { Std. error } \\
\text { kurtosis }\end{array}$ & $\begin{array}{c}\text { Shapiro- } \\
\text { Wilk W } \\
\text { Wilk } p\end{array}$ \\
\hline Perceived threat of COVID-19 & -0.49 & 0.06 & 0.37 & 0.12 & 0.98 \\
Aggressive tendencies during the pandemic & 0.46 & 0.06 & -0.41 & 0.12 & 0.97 \\
Sense of control during the pandemic & 0.05 & 0.06 & -0.48 & 0.12 & 0.99 \\
Sense of powerless during the pandemic & 0.28 & 0.06 & -0.26 & 0.12 & 0.99 \\
Positive coping during the pandemic & -0.33 & 0.06 & 0.18 & 0.12 & 0.99 \\
Negative coping during the pandemic & 0.21 & 0.06 & 0.26 & 0.12 & 0.99 \\
Life satisfaction during the pandemic & -0.17 & 0.06 & -0.30 & 0.12 & 0.99 \\
\hline
\end{tabular}

\section{Appendix 2}

Table 5 Mann-Whitney U test results for participants from different regions

\begin{tabular}{|c|c|c|c|c|c|c|c|c|c|}
\hline & \multirow[t]{2}{*}{ Participants in } & \multirow[t]{2}{*}{$\mathrm{N}$} & \multirow[t]{2}{*}{ Mean } & \multirow[t]{2}{*}{ Median } & \multirow[t]{2}{*}{$\mathrm{SD}$} & \multirow[t]{2}{*}{ SE } & \multicolumn{3}{|c|}{ Mann-Whitney U Test } \\
\hline & & & & & & & Statistic & $p$ & Effect Size \\
\hline \multirow[t]{2}{*}{ Perceived threat of COVID-19 } & Hubei Province & 757 & 3.60 & 3.62 & 0.66 & 0.02 & \multirow[t]{2}{*}{$240,421.00$} & \multirow[t]{2}{*}{$<0.001$} & \multirow[t]{2}{*}{0.21} \\
\hline & Other Regions of China & 799 & 3.34 & 3.38 & 0.68 & 0.02 & & & \\
\hline \multirow[t]{2}{*}{ Aggressive tendencies during the pandemic } & Hubei Province & 757 & 2.45 & 2.33 & 0.89 & 0.03 & \multirow[t]{2}{*}{$244,275.00$} & \multirow[t]{2}{*}{$<0.001$} & \multirow[t]{2}{*}{0.19} \\
\hline & Other Regions of China & 799 & 2.14 & 2.00 & 0.75 & 0.03 & & & \\
\hline \multirow[t]{2}{*}{ Sense of control during the pandemic } & Hubei Province & 757 & 2.95 & 2.92 & 0.84 & 0.03 & \multirow[t]{2}{*}{$224,367.50$} & \multirow[t]{2}{*}{$<0.001$} & \multirow[t]{2}{*}{0.26} \\
\hline & Other Regions of China & 799 & 3.33 & 3.33 & 0.82 & 0.03 & & & \\
\hline \multirow[t]{2}{*}{ Sense of powerless during the pandemic } & Hubei Province & 757 & 2.56 & 2.50 & 0.79 & 0.03 & \multirow[t]{2}{*}{$250,827.00$} & \multirow[t]{2}{*}{$<0.001$} & \multirow[t]{2}{*}{0.17} \\
\hline & Other Regions of China & 799 & 2.32 & 2.25 & 0.72 & 0.03 & & & \\
\hline \multirow[t]{2}{*}{ Positive coping during the pandemic } & Hubei Province & 757 & 3.58 & 3.67 & 0.64 & 0.02 & \multirow[t]{2}{*}{$250,238.50$} & \multirow[t]{2}{*}{$<0.001$} & \multirow[t]{2}{*}{0.17} \\
\hline & Other Regions of China & 799 & 3.76 & 3.83 & 0.58 & 0.02 & & & \\
\hline \multirow[t]{2}{*}{ Negative coping during the pandemic } & Hubei Province & 757 & 2.94 & 2.88 & 0.63 & 0.02 & \multirow[t]{2}{*}{$299,172.50$} & \multirow[t]{2}{*}{0.713} & \multirow[t]{2}{*}{0.01} \\
\hline & Other Regions of China & 799 & 2.94 & 3.00 & 0.61 & 0.02 & & & \\
\hline \multirow[t]{2}{*}{ Life satisfaction during the pandemic } & Hubei Province & 757 & 2.95 & 3.00 & 0.82 & 0.03 & \multirow[t]{2}{*}{$234,320.50$} & \multirow[t]{2}{*}{$<0.001$} & \multirow[t]{2}{*}{0.23} \\
\hline & Other Regions of China & 799 & 3.29 & 3.20 & 0.79 & 0.03 & & & \\
\hline
\end{tabular}

Supplementary Information The online version contains supplementary material available at https://doi.org/10.1007/s12144-021-01792-7.

Acknowledgments The authors thank the reviewers for their thoughtful comments on the early version of the manuscript.

Data Availability Statement (Data Transparency) The data generated during and/or analyzed during the current study are available from the corresponding author on reasonable request.

Code Availability (Software Application or Custom Code) The code for generating the study results is available from the corresponding author on reasonable request.
Authors' Contributions SD and XF jointly developed the concept and the design of the research, and jointly collected the data; SD analyzed the data; $\mathrm{SD}$ and $\mathrm{XF}$ jointly wrote the manuscript.

Funding The following grants funded this research: The Foundation of Humanities and Social Science of the Chinese Minister of Education (19YJC630027), Shanghai Foundation of Philosophy and Social Science (2017EGL006), and National Natural Science Foundation of China $(72042004,72002123)$.

\section{Declarations}

Conflicts of Interest/Competing Interests The authors declare that the research was conducted in the absence of any commercial or financial relationships that could be construed as a potential conflict of interest. 
Ethics Statement of the Survey This survey has been applied to and approved by the Yangtze University Research Ethics Committee in Psychology Department in March 2022, the reference number of the ethical approvement is 2020-03-23-2. In the survey, we gave written instructions to ensure the confidentiality and anonymity of the survey results. All study participants are free to choose whether to participate or not. We received the consent from all the study participants and followed the guidelines outlined in the Declaration of Helsinki.

\section{References}

Ahmed, M. Z., Ahmed, O., Aibao, Z., Hanbin, S., Siyu, L., \& Ahmad, A. (2020). Epidemic of COVID-19 in China and associated psychological problems. Asian Journal of Psychiatry, 51, 102092. https://doi. org/10.1016/j.ajp.2020.102092.

Allen, J. J., Anderson, C. A., \& Bushman, B. J. (2018). The general aggression model. Current Opinion in Psychology, 19, 75-80. https://doi.org/10.1016/j.copsyc.2017.03.034.

Berkowitz, L. (1989). Frustration-aggression hypothesis: Examination and reformulation. Psychological Bulletin, 106(1), 59-73. https:// doi.org/10.1037/0033-2909.106.1.59.

Biddlestone, M., Green, R., \& Douglas, K. M. (2020). Cultural orientation, power, belief in conspiracy theories, and intentions to reduce the spread of COVID-19. British Journal of Social Psychology, 59(3), 663-673. https://doi.org/10.1111/bjso.12397.

Bish, A., \& Michie, S. (2010). Demographic and attitudinal determinants of protective behaviours during a pandemic: A review. British Journal of Health Psychology, 15(4), 797-824. https://oi.org/10. 1348/135910710X485826.

Braga, C. G., \& Cruz, D. de A. L. M. da. (2009). Instrumento de Medida do Sentimento de Impotência para pacientes adultos. Revista Da Escola de Enfermagem Da USP, 43(spe), 1063-1070. https://doi. org/10.1590/S0080-62342009000500010

Brewer, N. T., Chapman, G. B., Gibbons, F. X., Gerrard, M., McCaul, K. D., \& Weinstein, N. D. (2007). Meta-analysis of the relationship between risk perception and health behavior: The example of vaccination. Health Psychology, 26(2), 136-145. https://doi.org/10. 1037/0278-6133.26.2.136

Brodsky, S. L. (2011). Hostility and scorn. In Therapy with coerced and reluctant clients. (pp. 141-155). American Psychological Association. https://doi.org/10.1037/12305-010.

Brown, M. J. (2001). Ethnic classification and culture: The case of the Tujia in Hubei, China. Asian Ethnicity, 2(1), 55-72. https://doi.org/ 10.1080/14631360120018013.

Brug, J., Aro, A. R., \& Richardus, J. H. (2009). Risk perceptions and behaviour: Towards pandemic control of emerging infectious diseases. International Journal of Behavioral Medicine, 16(1), 3-6. https://doi.org/10.1007/s12529-008-9000-x.

Censolo, R., \& Morelli, M. (2020). COVID-19 and the potential consequences for social stability. Peace Economics, Peace Science and Public Policy, 26(3), 1-5. https://doi.org/10.1515/peps-2020-0045.

Dempsey, M. (2002). Negative coping as mediator in the relation between violence and outcomes: Inner-city African American youth. American Journal of Orthopsychiatry, 72(1), 102-109. https://doi. org/10.1037/0002-9432.72.1.102.

Deng, S., Wang, W., Xie, P., Chao, Y., \& Zhu, J. (2020). Perceived severity of COVID-19 and post-pandemic consumption willingness: The roles of boredom and sensation-seeking. Frontiers in Psychology, 11, 1-10. https://doi.org/10.3389/fpsyg.2020.567784.

Diamond, P. M., \& Magaletta, P. R. (2006). The short-form buss-Perry aggression questionnaire (BPAQ-SF). Assessment, 13(3), 227-240. https://doi.org/10.1177/1073191106287666.

Dryhurst, S., Schneider, C. R., Kerr, J., Freeman, A. L. J., Recchia, G., van der Bles, A. M., Spiegelhalter, D., \& van der Linden, S. (2020).
Risk perceptions of COVID-19 around the world. Journal of Risk Research, 23(7-8), 994-1006. https://doi.org/10.1080/13669877. 2020.1758193 .

Faulkner, J., Schaller, M., Park, J. H., \& Duncan, L. A. (2004). Evolved disease-avoidance mechanisms and contemporary xenophobic attitudes. Group Processes \& Intergroup Relations, 7(4), 333-353. https://doi.org/10.1177/1368430204046142.

Folkman, S., \& Lazarus, R. S. (1988). The relationship between coping and emotion: Implications for theory and research. Social Science \& Medicine, 26(3), 309-317. https://doi.org/10.1016/0277-9536(88) 90395-4.

Gao, Y., Sun, F., Jiang, W., Fang, Y., Yue, L., Lin, X., \& Li, X. (2020). Beliefs towards the COVID-19 pandemic among patients with emotional disorders in China. General Psychiatry, 33(3), e100231. https://doi.org/10.1136/gpsych-2020-100231.

Global Times. (2020). Chinese families see surge in domestic violence amid COVID-19 lockdown. https://www.globaltimes.cn/content/ 1182484.shtml

Guo, Q., Zheng, Y., Shi, J., Wang, J., Li, G., Li, C., Fromson, J. A., Xu, Y., Liu, X., Xu, H., Zhang, T., Lu, Y., Chen, X., Hu, H., Tang, Y., Yang, S., Zhou, H., Wang, X., Chen, H., Wang, Z., \& Yang, Z. (2020). Immediate psychological distress in quarantined patients with COVID-19 and its association with peripheral inflammation: A mixed-method study. Brain, Behavior, and Immunity, 88, 17-27. https://doi.org/10.1016/j.bbi.2020.05.038.

Guterres, A. (2020). Global cooperation must adapt to meet biggest threat since second world war, secretary-general says on international day, as COVID-19 transcends Borders. United Nations. https://www.un.org/press/en/2020/sgsm20058.doc.htm

Hayes, A. F. (2013). Introduction to mediation, moderation, and conditional process analysis (1st ed.). Guilford Press.

Hu, L., \& Bentler, P. M. (1999). Cutoff criteria for fit indexes in covariance structure analysis: Conventional criteria versus new alternatives. Structural Equation Modeling: A Multidisciplinary Journal, 6(1), 1-55. https://doi.org/10.1080/10705519909540118.

Kim, H. S., Sherman, D. K., \& Updegraff, J. A. (2016). Fear of Ebola. Psychological Science, 27(7), 935-944. https://doi.org/10.1177/ 0956797616642596.

Kim, J. (2020). Impact of the perceived threat of COVID-19 on varietyseeking. Australasian Marketing Journal (AMJ)., 28, 108-116. https://doi.org/10.1016/j.ausmj.2020.07.001.

Lachman, M. E., \& Weaver, S. L. (1998). The sense of control as a moderator of social class differences in health and well-being. Journal of Personality and Social Psychology, 74(3), 763-773. https://doi.org/10.1037/0022-3514.74.3.763.

Li, C.-H. (2016). The performance of ML, DWLS, and ULS estimation with robust corrections in structural equation models with ordinal variables. Psychological Methods, 21(3), 369-387. https://doi.org/ 10.1037/met0000093.

Li, J.-B., Yang, A., Dou, K., Wang, L.-X., Zhang, M.-C., \& Lin, X.-Q. (2020). Chinese Public's knowledge, perceived severity, and perceived controllability of COVID-19 and their associations with emotional and behavioural reactions, social participation, and precautionary behaviour: A National Survey. BMC Public Health, Preprint, 1-18. https://doi.org/10.21203/rs.3.rs-16572/v4.

Lin, C.-Y. (2020). Social reaction toward the 2019 novel coronavirus (COVID-19). Social Health and Behavior, 3(1), 1. https://doi.org/ 10.4103/SHB.SHB 11 20.

Liu, Q., Luo, D., Haase, J. E., Guo, Q., Wang, X. Q., Liu, S., Xia, L., Liu, Z., Yang, J., \& Yang, B. X. (2020). The experiences of health-care providers during the COVID-19 crisis in China: A qualitative study. The Lancet Global Health, 8(6), e790-e798. https://doi.org/10. 1016/S2214-109X(20)30204-7.

Liu, S., Lithopoulos, A., Zhang, C.-Q., Garcia-Barrera, M. A., \& Rhodes, R. E. (2021). Personality and perceived stress during COVID-19 pandemic: Testing the mediating role of perceived threat and 
efficacy. Personality and Individual Differences, 168, 110351. https://doi.org/10.1016/j.paid.2020.110351.

Lu, P., \& Liu, F. (2020). Research on epidemic risk cognitive process and intergenerational interaction model (in Chinese). Journal of Central South University (Social Sciences), 3, 11-23. https://doi.org/10. 11817/j.issn.1672-3104.2020.03.002.

MacDonald, J. M., Piquero, A. R., Valois, R. F., \& Zullig, K. J. (2005). The relationship between life satisfaction, risk-taking behaviors, and youth violence. Journal of Interpersonal Violence, 20(11), 14951518. https://doi.org/10.1177/0886260505278718.

Mariani, R., Renzi, A., Di Trani, M., Trabucchi, G., Danskin, K., \& Tambelli, R. (2020). The impact of coping strategies and perceived family support on depressive and anxious symptomatology during the coronavirus pandemic (COVID-19) lockdown. Frontiers in Psychiatry, 11, 1-9. https://doi.org/10.3389/fpsyt.2020.587724.

Maxwell, J. P., \& Siu, O. L. (2008). The Chinese coping strategies scale: Relationships with aggression, anger, and rumination in a diverse sample of Hong Kong Chinese adults. Personality and Individual Differences, 44(5), 1049-1059. https://doi.org/10.1016/j.paid.2007. 10.006 .

Myers, R. H., \& Montgomery, D. C. (1997). A tutorial on generalized linear models. Journal of Quality Technology, 29(3), 274-291. https://doi.org/10.1080/00224065.1997.11979769.

Navarrete, C. D., \& Fessler, D. M. T. (2006). Disease avoidance and ethnocentrism: The effects of disease vulnerability and disgust sensitivity on intergroup attitudes. Evolution and Human Behavior, 27(4), 270-282. https://doi.org/10.1016/j.evolhumbehav.2005.12. 001 .

O'Shea, B. A., Watson, D. G., Brown, G. D. A., \& Fincher, C. L. (2020). Infectious disease prevalence, not race exposure, predicts both implicit and explicit racial prejudice across the United States. Social Psychological and Personality Science, 11(3), 345-355. https://doi. org/10.1177/1948550619862319.

Ogueji, I. A., Okoloba, M. M., \& Demoko Ceccaldi, B. M. (2021). Coping strategies of individuals in the United Kingdom during the COVID-19 pandemic. Current Psychology, online, 1-7. https://doi. org/10.1007/s12144-020-01318-7.

Orzechowska, A., Florkowski, A., Gruszczyński, W., Zboralski, K., Wysokiński, A., Gałecki, P., \& Talarowska, M. (2009). Socioeconomic status, aggressive behaviours and coping with stress. Psychiatria Polska, 43(1), 53-63 http://www.ncbi.nlm.nih.gov/ pubmed/19694400.

Park, J. H., Faulkner, J., \& Schaller, M. (2003). Evolved diseaseavoidance processes and contemporary anti-social behavior: Prejudicial attitudes and avoidance of people with physical disabilities. Journal of Nonverbal Behavior, 27, 65-87. https://doi.org/10. 1023/A:1023910408854

Parmet, W. E., \& Sinha, M. S. (2017). A panic foretold: Ebola in the United States. Critical Public Health, 27(1), 148-155. https://doi. org/10.1080/09581596.2016.1159285.

Pavot, W., \& Diener, E. (1993). Review of the satisfaction with life scale. Psychological Assessment, 5(2), 164-172. https://doi.org/10.1037/ 1040-3590.5.2.164

Poon, K.-T., Chen, Z., Teng, F., \& Wong, W.-Y. (2020). The effect of objectification on aggression. Journal of Experimental Social Psychology, 87, 103940. https://doi.org/10.1016/j.jesp.2019. 103940.

Qiu, J., Shen, B., Zhao, M., Wang, Z., Xie, B., \& Xu, Y. (2020). A nationwide survey of psychological distress among Chinese people in the COVID-19 epidemic: Implications and policy recommendations. General Psychiatry, 33(2), e100213. https://doi.org/10.1136/ gpsych-2020-100213.

Reich, J. W., \& Infurna, F. J. (2017). Perceived control: Theory, research, and practice in the first 50 years. Oxford University Press.
Rosseel, Y. (2012). Lavaan: An R package for structural equation modeling. Journal of Statistical Software, 48(2), 1-36 https://www. jstatsoft.org/v48/i02/.

Schaller, M., \& Park, J. H. (2011). The behavioral immune system (and why it matters). Current Directions in Psychological Science, 20(2), 99-103. https://doi.org/10.1177/0963721411402596.

Schat, A. C. H., \& Kelloway, E. K. (2000). Effects of perceived control on the outcomes of workplace aggression and violence. Journal of Occupational Health Psychology, 5(3), 386-402. https://doi.org/10. 1037/1076-8998.5.3.386.

Sherrod, D. R., Moore, B. S., \& Underwood, B. (1979). Environmental noise, perceived control, and aggression. The Journal of Social Psychology, 109(2), 245-252. https://doi.org/10.1080/00224545. 1979.9924200

Shi, D., \& Maydeu-Olivares, A. (2020). The effect of estimation methods on SEM fit indices. Educational and Psychological Measurement, 80(3), 421-445. https://doi.org/10.1177/0013164419885164.

Skinner, E. A., \& Zimmer-Gembeck, M. (2016). Coping. In H. S. Friedman (Ed.), Encyclopedia of Mental Health (pp. 350-357). Elsevier. https://doi.org/10.1016/B978-0-12-397045-9.00036-7.

Suldo, S. M., \& Huebner, E. S. (2004). Does life satisfaction moderate the effects of stressful life events on psychopathological behavior during adolescence? School Psychology Quarterly, 19(2), 93-105. https://doi.org/10.1521/scpq.19.2.93.33313.

Terrizzi, J. A., Shook, N. J., \& McDaniel, M. A. (2013). The behavioral immune system and social conservatism: A meta-analysis. Evolution and Human Behavior, 34(2), 99-108. https://doi.org/10. 1016/j.evolhumbehav.2012.10.003.

The jamovi project. (2021). jamovi (v1.6). https://www.jamovi.org

Tong, H. (2004). Model of SARS stress and It's character (in Chinese). Acta Psychologica Sinica, 36(1), 103-109 http://journal.psych.ac. $\mathrm{cn} / \mathrm{xlxb} / \mathrm{CN} / \mathrm{Y} 2004 / \mathrm{V} 36 / \mathrm{I01} / 103$.

Valois, R. F., Zullig, K. J., Huebner, E. S., \& Drane, J. W. (2001). Relationship between life satisfaction and violent behaviors among adolescents. American Journal of Health Behavior, 25(4), 353-366. https://doi.org/10.5993/AJHB.25.4.1.

Velotti, P., Garofalo, C., Bottazzi, F., \& Caretti, V. (2017). Faces of shame: Implications for self-esteem, emotion regulation, aggression, and well-being. The Journal of Psychology, 151(2), 171-184. https://doi.org/10.1080/00223980.2016.1248809.

Wang, C., Pan, R., Wan, X., Tan, Y., Xu, L., McIntyre, R. S., Choo, F. N., Tran, B., Ho, R., Sharma, V. K., \& Ho, C. (2020). A longitudinal study on the mental health of general population during the COVID19 epidemic in China. Brain, Behavior, and Immunity, 87, 40-48. https://doi.org/10.1016/j.bbi.2020.04.028.

Warburton, W. A., McIlwain, D. J. F., Cairns, D. R., \& Taylor, A. J. (2016). Restoring perceived control with aggression: Why, how and who? World Meeting of the International Society for Research on Aggression. https://researchers.mq.edu.au/en/publications/ restoring-perceived-control-with-aggression-why-how-and-who

Wasil, A. R., Franzen, R. E., Gillespie, S., Steinberg, J. S., Malhotra, T., \& DeRubeis, R. J. (2021). Commonly reported problems and coping strategies during the COVID-19 crisis: A survey of graduate and professional students. Frontiers in Psychology, 12, 1-14. https:// doi.org/10.3389/fpsyg.2021.598557.

Wen, F., Ma, S., Ye, H., Qi, Y., \& Zuo, B. (2020). "Psychological typhoon eye effect" and "ripple effect": Double perspective test of risk perception and anxiety characteristics of people in different COVID19 severity regions (in Chinese). Acta Psychologica Sinica, 52(9), 1087. https://doi.org/10.3724/SP.J.1041.2020.01087.

Xia, Y., \& Yang, Y. (2019). RMSEA, CFI, and TLI in structural equation modeling with ordered categorical data: The story they tell depends on the estimation methods. Behavior Research Methods, 51(1), 409-428. https://doi.org/10.3758/s13428-018-1055-2. 
Xie, Y. (1998). Reliability and validity of the simplified coping style questionnaire (in Chinese). Chinese Journal of Clinical Psychology, 6(2), 114-115.

Xin, M., Luo, S., She, R., Yu, Y., Li, L., Wang, S., Ma, L., Tao, F., Zhang, J., Zhao, J., Li, L., Hu, D., Zhang, G., Gu, J., Lin, D., Wang, H., Cai, Y., Wang, Z., You, H., Hu, G., \& Lau, J. T. (2020). Negative cognitive and psychological correlates of mandatory quarantine during the initial COVID-19 outbreak in China. American Psychologist, 75(5), 607-617. https://doi.org/10.1037/ amp0000692.

Xin, Z. (2020). Public perception of pandemic risk and economic confidence survey (in Chinese). People's Forum, 5, 32-35 http://www. rmlt.com.cn/2020/0226/570221.shtml.

Xinhua News Agency. (2020). Fighting COVID-19: China in Action. http://www.xinhuanet.com/english/2020-06/07/c_139120424.htm

Ye, B., Zeng, Y., Hohjin Im, Liu, M., Wang, X., \& Yang, Q. (2020). The relationship between fear of COVID-19 and online aggressive behavior: A moderated mediation model. PsyArXiv Preprints. https:// doi.org/10.31234/osf.io/kym49

Ying, Y., Ruan, L., Kong, F., Zhu, B., Ji, Y., \& Lou, Z. (2020). Mental health status among family members of health care workers in Ningbo, China, during the coronavirus disease 2019 (COVID-19) outbreak: A cross-sectional study. BMC Psychiatry, 20(1), 379. https://doi.org/10.1186/s12888-020-02784-w.
Zhang, L., Ma, M., Li, D., \& Xin, Z. (2020a). The psychological typhoon eye effect during the COVID-19 outbreak in China: The role of coping efficacy and perceived threat. Globalization and Health, 16(1), 105. https://doi.org/10.1186/s12992-020-00626-8.

Zhang, S. X., Huang, H., \& Wei, F. (2020b). Geographical distance to the epicenter of Covid-19 predicts the burnout of the working population: Ripple effect or typhoon eye effect? Psychiatry Research, 288, 112998. https://doi.org/10.1016/j.psychres.2020.112998.

Zhang, S. X., Wang, Y., Rauch, A., \& Wei, F. (2020c). Unprecedented disruption of lives and work: Health, distress and life satisfaction of working adults in China one month into the COVID-19 outbreak. Psychiatry Research, 288, 112958. https://doi.org/10.1016/j. psychres.2020.112958.

Zhong, Y., Liu, W., Lee, T.-Y., Zhao, H., \& Ji, J. (2020). Risk perception, knowledge, information sources and emotional states among COVID-19 patients in Wuhan, China: A Cross-sectional Survey. Nursing Outlook, 69(1), 13-21. https://doi.org/10.1016/j.outlook. 2020.08.005.

Publisher's Note Springer Nature remains neutral with regard to jurisdictional claims in published maps and institutional affiliations. 